\title{
Classification of integrable $(2+1)$-dimensional quasilinear hierarchies
}

\author{
E.V. Ferapontov*, K.R. Khusnutdinova** and M.V. Pavlov \\ Department of Mathematical Sciences \\ Loughborough University \\ Loughborough, Leicestershire LE11 3TU \\ United Kingdom \\ e-mails: \\ E.V.Ferapontov@lboro.ac.uk \\ K.Khusnutdinova@lboro.ac.uk \\ M.V.Pavlov@lboro.ac.uk
}

\begin{abstract}
We investigate (2+1)-dimensional hierarchies associated with integrable PDE's of the form

$$
\Omega_{t t}=F\left(\Omega_{x x}, \Omega_{x t}, \Omega_{x y}\right),
$$

which generalize the dispersionless KP hierarchy. The integrability is understood as the existence of infinitely many hydrodynamic reductions.
\end{abstract}

MSC: 35Q58, 37K05, 37K10.

Keywords: Integrable hierachies of dKP type, Hydrodynamic Reductions, Pseudopotentials.

${ }^{*}$ Center for Nonlinear Studies, Landau Institute for Theoretical Physics, Kosygina 2, Moscow, 117940, Russia.

** On the leave from: Institute of Mechanics, Ufa Branch of the Russian Academy of Sciences, Karl Marx Str. 6, Ufa, 450000, Russia. 


\section{Introduction}

Let us consider a function $\Omega$ of infinitely many independent variables $t^{0}, t^{1}, t^{2}, \ldots$ satisfying a system of second order PDE's

$$
\Omega_{n k}=\Phi_{n k}\left(\Omega_{00}, \Omega_{01}, \Omega_{02}, \ldots, \Omega_{0, n+k}\right) ;
$$

here $\Omega_{n k} \equiv \partial_{t^{n}} \partial_{t^{k}} \Omega, \quad n \geqslant 1, k \geqslant 1$. Explicitly, one has

$$
\begin{aligned}
& \Omega_{11}=\Phi_{11}\left(\Omega_{00}, \Omega_{01}, \Omega_{02}\right), \\
& \Omega_{12}=\Phi_{12}\left(\Omega_{00}, \Omega_{01}, \Omega_{02}, \Omega_{03}\right), \\
& \Omega_{13}=\Phi_{13}\left(\Omega_{00}, \Omega_{01}, \Omega_{02}, \Omega_{03}, \Omega_{04}\right), \\
& \Omega_{22}=\Phi_{22}\left(\Omega_{00}, \Omega_{01}, \Omega_{02}, \Omega_{03}, \Omega_{04}\right),
\end{aligned}
$$

etc. Equations of this type generalize the dispersionless KP hierarchy

$$
\begin{aligned}
\Omega_{11} & =\Omega_{02}-\frac{1}{2} \Omega_{00}^{2} \\
\Omega_{12} & =\Omega_{03}-\Omega_{00} \Omega_{01} \\
\Omega_{13} & =\Omega_{04}-\Omega_{00} \Omega_{02}-\frac{1}{2} \Omega_{01}^{2} \\
\Omega_{22} & =\Omega_{04}+\frac{1}{3} \Omega_{00}^{3}-\Omega_{00} \Omega_{02}-\Omega_{01}^{2}
\end{aligned}
$$

etc. Further examples arise in the theory of Dirichlet's problem in multi-connected domains [8]. The compatibility conditions of the equations (1) impose strong restrictions on the functions $\Phi_{n k}$ implying, in particular, that $\Phi_{11}$ uniquely determines the rest of the functions $\Phi_{n k}[10],[2]$. The function $\Phi_{11}$ itself satisfies a complicated over-determined system of third order PDE's (see Sect. 2 where we re-derive this system based on the method of hydrodynamic reductions [7], [4], [6], [9]). Its general solution can be reduced to either of the four essentially different canonical forms

$$
\begin{aligned}
\Phi_{11} & =\Omega_{02}+\frac{1}{4 A}\left(A \Omega_{01}+2 B \Omega_{00}\right)^{2}+C e^{-A \Omega_{00}}, \\
\Phi_{11} & =\frac{\Omega_{02}}{\Omega_{00}}+\left(\frac{1}{\Omega_{00}}+\frac{A}{4 \Omega_{00}^{2}}\right) \Omega_{01}^{2}+\frac{B}{\Omega_{00}^{2}} \Omega_{01}+\frac{B^{2}}{A \Omega_{00}^{2}}+C e^{A / \Omega_{00}}, \\
\Phi_{11} & =\frac{\Omega_{02}}{\Omega_{01}}+\frac{1}{6} \eta\left(\Omega_{00}\right) \Omega_{01}^{2}, \\
\Phi_{11} & =\ln \Omega_{02}-\ln \theta_{1}\left(\Omega_{01}, \Omega_{00}\right)-\frac{1}{4} \int \eta(\tau) d \tau,
\end{aligned}
$$

see $[\mathbf{1 0}]$. Here $\eta(\tau)$ is a solution of the Chazy equation

$$
\eta^{\prime \prime \prime}+2 \eta \eta^{\prime \prime}=3 \eta^{\prime 2}
$$

which can be represented in parametric form

$$
\eta(\tau)=\frac{4}{\pi^{2}} \mathbf{K}(s)\left[\left(2-s^{2}\right) \mathbf{K}(s)-3 \mathbf{E}(s)\right], \quad \tau=-\pi^{2} \frac{\mathbf{K}\left(\sqrt{1-s^{2}}\right)}{\mathbf{K}(s)},
$$


where $\mathbf{K}(s)$ and $\mathbf{E}(s)$ are complete elliptic integrals of the first and second kind, respectively [1]. The theta-function

$$
\theta_{1}(z, \tau)=2 \sum_{n=0}^{\infty}(-1)^{n} e^{-(n+1 / 2)^{2} \tau} \sin [(2 n+1) z]
$$

is defined as a solution of the involutive system

$$
\begin{gathered}
\partial_{z} \theta_{1}=-k \theta_{1}, \quad \partial_{\tau} \theta_{1}=\frac{1}{4}\left(k^{2}-l\right) \theta_{1}, \\
\partial_{z} k=l, \quad \partial_{\tau} k=\frac{1}{4} \sqrt{4 l^{3}-4 \eta l^{2}-8 \eta^{\prime} l-\frac{8}{3} \eta^{\prime \prime}}-\frac{1}{2} k l, \\
\partial_{z} l=\sqrt{4 l^{3}-4 \eta l^{2}-8 \eta^{\prime} l-\frac{8}{3} \eta^{\prime \prime}}, \quad \partial_{\tau} l=l^{2}-\eta l-\eta^{\prime}-\frac{1}{2} k \sqrt{4 l^{3}-4 \eta l^{2}-8 \eta^{\prime} l-\frac{8}{3} \eta^{\prime \prime}},
\end{gathered}
$$

where, again, $\eta$ solves the Chazy equation (2). We emphasize, however, that one does not need the explicit formulae for $\theta_{1}$ and $\eta$ to work with the above expressions for $\Phi_{11}$ : what one actually needs are the equations (2), (3).

The dKP hierarhy corresponds to a simple degeneration of the first canonical form: $A=-2 B^{2}, B \rightarrow 0, C=0$. Similarly, the hierarchy of the modified dKP equation,

$$
\Omega_{11}=\Omega_{02}+B \Omega_{00} \Omega_{01}+\frac{B^{2}}{3} \Omega_{00}^{3}
$$

can be obtained by the degeneration $C=-2 B^{2} A^{-3}, A \rightarrow 0$ (along with an appropriate linear change of the variable $t^{2}$ ).

In Sect. 2 we concentrate on the first equation

$$
\Omega_{11}=\Phi_{11}\left(\Omega_{00}, \Omega_{01}, \Omega_{02}\right),
$$

dropping any assumptions on the structure of higher flows of the hierarchy. Introducing the notation $t^{0} \equiv x, t^{1} \equiv t, t^{2} \equiv y, \Phi_{11} \equiv G, \Omega_{00} \equiv a, \Omega_{01} \equiv b, \Omega_{02} \equiv c$ we rewrite (4) in a quasilinear form

$$
a_{t}=b_{x}, a_{y}=c_{x}, b_{y}=c_{t}, b_{t}=G(a, b, c)_{x} .
$$

Applying to (5) the method of hydrodynamic reductions (as outlined in [6]), we arrive at the same system of PDE's for $\Phi_{11}$ as the one obtained in [10]. This confirms that the symmetry approach of [10] based on equations (1) yields a complete list of integrable PDE's of the form (4).

In Sect. 3 we discuss scalar pseudopotentials

$$
\psi_{t}=Q\left(\psi_{x}, \Omega_{00}, \Omega_{01}\right), \psi_{y}=L\left(\psi_{x}, \Omega_{00}, \Omega_{01}, \Omega_{02}\right)
$$

which play a role of dispersionless Lax pairs [12] for equations (4). To calculate pseudopotentials we introduce negative times $t^{-1}, t^{-2}, \ldots$ and consider the corresponding negative flows of the hierarchy.

\section{Classification of integrable PDEs of the form $\Omega_{t t}=G\left(\Omega_{x x}, \Omega_{x t}, \Omega_{x y}\right)$}

In this section we demonstrate how the classification results of [10] (see also [2]) follow from the method of hydrodynamic reductions as proposed in [6]. Introducing the notation $\Omega_{x x}=a, \Omega_{x t}=b, \Omega_{x y}=c, \Omega_{t t}=G(a, b, c)$ we first rewrite our PDE in the quasilinear 
form (5). Looking for hydrodynamic reductions in the form $a=a\left(R^{1}, \ldots, R^{n}\right), b=$ $b\left(R^{1}, \ldots, R^{n}\right), c=c\left(R^{1}, \ldots, R^{n}\right)$ where the Riemann invariants satisfy the equations

$$
R_{t}^{i}=\lambda^{i}(R) R_{x}^{i}, \quad R_{y}^{i}=\mu^{i}(R) R_{x}^{i}
$$

and substituting into (5), one arrives at

$$
\partial_{i} b=\lambda^{i} \partial_{i} a, \partial_{i} c=\mu^{i} \partial_{i} a
$$

along with the dispersion relation

$$
\left(\lambda^{i}\right)^{2}=G_{a}+G_{b} \lambda^{i}+G_{c} \mu^{i} .
$$

The commutativity conditions of the flows (6) are of the form

$$
\frac{\partial_{j} \lambda^{i}}{\lambda^{j}-\lambda^{i}}=\frac{\partial_{j} \mu^{i}}{\mu^{j}-\mu^{i}}, i \neq j, \partial_{j}=\partial / \partial_{R^{j}}
$$

see [11]. Differentiating the dispersion relation and taking into account (7) one obtains the expressions for $\partial_{j} \lambda^{i}$ in the form

$$
\begin{gathered}
\partial_{j} \lambda^{i}=\frac{\partial_{j} a}{G_{c}\left(\lambda^{i}-\lambda^{j}\right)}\left(G_{a a} G_{c}+G_{a b} G_{c}\left(\lambda^{i}+\lambda^{j}\right)+G_{b b} G_{c} \lambda^{i} \lambda^{j}+\right. \\
\left(G_{a c}+\lambda^{i} G_{b c}\right)\left(\left(\lambda^{j}\right)^{2}-G_{b} \lambda^{j}-G_{a}\right) \\
\left.+\left(\left(\lambda^{i}\right)^{2}-G_{b} \lambda^{i}-G_{a}\right)\left[G_{a c}+G_{b c} \lambda^{j}+\frac{G_{c c}}{G_{c}}\left(\left(\lambda^{j}\right)^{2}-G_{b} \lambda^{j}-G_{a}\right)\right]\right) .
\end{gathered}
$$

The compatibility conditions of the equations $\partial_{i} b=\lambda^{i} \partial_{i} a$ and $\partial_{i} c=\mu^{i} \partial_{i} a$ imply

$$
\partial_{i} \partial_{j} a=\frac{\partial_{j} \lambda^{i}}{\lambda^{j}-\lambda^{i}} \partial_{i} a+\frac{\partial_{i} \lambda^{j}}{\lambda^{i}-\lambda^{j}} \partial_{j} a
$$

One can see that the consistency conditions of the equations (8), that is, $\partial_{k} \partial_{j} \lambda^{i}-\partial_{j} \partial_{k} \lambda^{i}=$ 0 , are of the form $P \partial_{j} a \partial_{k} a=0$ where $P$ is a complicated rational expression in $\lambda^{i}, \lambda^{j}, \lambda^{k}$ whose coefficients depend on partial derivatives of $G(a, b, c)$ up to third order (to obtain the integrability conditions it suffices to consider 3-component reductions setting $i=1, j=$ $2, k=3)$. Requiring that $P$ vanishes identically we obtain the expressions for all third order partial derivatives of $G$. Similarly, the compatibility conditions of the equations (9), that is, $\partial_{k}\left(\partial_{i} \partial_{j} a\right)-\partial_{j}\left(\partial_{i} \partial_{k} a\right)=0$, take the form $S \partial_{i} a \partial_{j} a \partial_{k} a=0$ where, again, $S$ is rational in $\lambda^{i}, \lambda^{j}, \lambda^{k}$. Equating $S$ to zero one obtains exactly the same conditions as the ones obtained on the previous step. The resulting set of integrability conditions looks as follows: 


$$
\begin{aligned}
G_{c c c} & =\frac{2 G_{c c}^{2}}{G_{c}}, \quad G_{a c c}=\frac{2 G_{a c} G_{c c}}{G_{c}}, \quad G_{b c c}=\frac{2 G_{b c} G_{c c}}{G_{c}}, \\
G_{a a c} & =\frac{2 G_{a c}^{2}}{G_{c}}, \quad G_{a b c}=\frac{2 G_{a c} G_{b c}}{G_{c}}, \quad G_{b b c}=\frac{2 G_{b c}^{2}}{G_{c}}, \\
G_{b b b} & =\frac{2}{G_{c}^{2}}\left(G_{b} G_{b c}^{2}+G_{b c}\left(G_{c} G_{b b}+2 G_{a c}\right)-G_{c c}\left(G_{b} G_{b b}+2 G_{a b}\right)\right), \\
G_{a b b} & =\frac{2}{G_{c}^{2}}\left(G_{a} G_{b c}^{2}+G_{a c}\left(G_{c} G_{b b}+G_{a c}\right)-G_{c c}\left(G_{a} G_{b b}+G_{a a}\right)\right), \\
G_{a a b} & =\frac{2}{G_{c}^{2}}\left(G_{c c}\left(G_{b} G_{a a}-2 G_{a} G_{a b}\right)-G_{a c}\left(G_{b} G_{a c}-2 G_{c} G_{a b}\right)-G_{b c}\left(G_{c} G_{a a}-2 G_{a} G_{a c}\right)\right), \\
G_{a a a} & =\frac{2}{G_{c}^{2}}\left(\left(G_{a}+G_{b}^{2}\right) G_{a c}^{2}+G_{a}^{2} G_{b c}^{2}+G_{c}^{2}\left(G_{a b}^{2}-G_{a a} G_{b b}\right)\right. \\
& +G_{a c} G_{c}\left(G_{a a}+2\left(G_{a} G_{b b}-G_{b} G_{a b}\right)\right)+2 G_{b c}\left(G_{b}\left(G_{c} G_{a a}-G_{a} G_{a c}\right)-G_{a} G_{c} G_{a b}\right) \\
& \left.-G_{c c}\left(\left(G_{a}+G_{b}^{2}\right) G_{a a}-2 G_{a} G_{b} G_{a b}\right)\right) .
\end{aligned}
$$

This system is in involution and its general solution depends on 10 integration constants (indeed, the values of $G$ and its partial derivatives up to second order can be prescribed arbitrarily at any point $\left.a_{0}, b_{0}, c_{0}\right)$.

The integration of the first six equations in (10) yields

$$
G(a, b, c)=\frac{1}{\varepsilon} \ln (\alpha a+\beta b+\gamma+\varepsilon c)+F(a, b) .
$$

The substitution of this ansatz into the remaining equations imposes further constraints on the function $F(a, b)$,

$$
\begin{gathered}
F_{b b b}-4 \varepsilon F_{a b}-2 \varepsilon F_{b} F_{b b}=0, \\
F_{a b b}-2 \varepsilon F_{a a}-2 \varepsilon F_{a} F_{b b}=0, \\
F_{a a b}+2 \varepsilon F_{b} F_{a a}-4 \varepsilon F_{a} F_{a b}=0 \\
F_{a a a}-2 \varepsilon F_{a} F_{a a}+2 F_{a a} F_{b b}-2 \varepsilon F_{b}^{2} F_{a a}-2 F_{a b}^{2}+4 \varepsilon F_{a} F_{b} F_{a b}-2 \varepsilon F_{a}^{2} F_{b b}=0,
\end{gathered}
$$

which identically coincide with the ones derived in [10]. The first equation in (11) has the general solution

$$
F=-\frac{1}{4 \varepsilon} \int \eta(a) d a-\frac{1}{\varepsilon} \ln \theta(a, b), \quad 4 \varepsilon \theta_{a}=\theta_{b b},
$$

and the substitution of (12) into the remaining equations (11) and further integration lead to the four essentially different cases as shown in [10].

This confirms that the method of higher symmetries adopted in [10] gives all integrable hierarchies of the form (1).

\section{Pseudopotentials}

Let us introduce the negative times $t^{-1}, t^{-2}, t^{-3}, \ldots$ and extend the hierarchy (1) by the equations

$$
\begin{aligned}
\Omega_{n,-k} & =\Phi_{n,-k}\left(\Omega_{0,-k}, \Omega_{0,-k+1}, \ldots, \Omega_{00}, \Omega_{01}, \ldots, \Omega_{0 n}\right) \\
\Omega_{-n,-k} & =\Phi_{-n,-k}\left(\Omega_{0,-n-k}, \Omega_{0,-n-k+1}, \ldots, \Omega_{00}\right)
\end{aligned}
$$


in particular,

$$
\begin{aligned}
& \Omega_{1,-1}=\Phi_{1,-1}\left(\Omega_{0,-1}, \Omega_{00}, \Omega_{01}\right), \\
& \Omega_{2,-1}=\Phi_{2,-1}\left(\Omega_{0,-1}, \Omega_{00}, \Omega_{01}, \Omega_{02}\right) .
\end{aligned}
$$

In the notation $t^{0} \equiv x, t^{1} \equiv t, t^{2} \equiv y, t^{-1} \equiv z, \Phi_{1,-1} \equiv Q, \Phi_{2,-1} \equiv L$ we can rewrite these equations in the form

$$
\begin{aligned}
& \Omega_{t z}=Q\left(\Omega_{x z}, \Omega_{x x}, \Omega_{x t}\right), \\
& \Omega_{y z}=L\left(\Omega_{x z}, \Omega_{x x}, \Omega_{x t}, \Omega_{x y}\right) .
\end{aligned}
$$

In terms of $\psi=\Omega_{z}$ this provides a pseudopotential

$$
\begin{aligned}
\psi_{t} & =Q\left(\psi_{x}, \Omega_{x x}, \Omega_{x t}\right), \\
\psi_{y} & =L\left(\psi_{x}, \Omega_{x x}, \Omega_{x t}, \Omega_{x y}\right)
\end{aligned}
$$

for the equation (4). Below we demonstrate how one can obtain explicit expressions for both functions $Q$ and $L$.

Remark: The hierarchy of the Boyer-Finley equation [3]

$$
\Omega_{1,-1}=\exp \Omega_{00}
$$

can be obtained by a simple degeneration $B=C=0, A=-2$ in the first canonical form; the first commuting flow of this hierarchy is

$$
\Omega_{11}=\Omega_{02}-\frac{1}{2} \Omega_{01}^{2}
$$

Let us first derive the explicit form for the function $Q$ applying the method of hydrodynamic reductions to the PDE

$$
\Omega_{z t}=Q\left(\Omega_{x z}, \Omega_{x x}, \Omega_{x t}\right) .
$$

Introducing the notation $\Omega_{x x}=a, \Omega_{x t}=b, \Omega_{x z}=e, \Omega_{z t}=Q(e, a, b)$, one can rewrite this PDE in the quasilinear form

$$
a_{t}=b_{x}, a_{z}=e_{x}, b_{z}=e_{t}=Q(e, a, b)_{x} .
$$

Looking for reductions in the form $a=a\left(R^{1}, \ldots, R^{n}\right), b=b\left(R^{1}, \ldots, R^{n}\right), e=e\left(R^{1}, \ldots, R^{n}\right)$ where the Riemann invariants satisfy the equations

$$
R_{t}^{i}=\lambda^{i}(R) R_{x}^{i}, \quad R_{z}^{i}=\zeta^{i}(R) R_{x}^{i}
$$

and substituting into (13), one arrives at

$$
\partial_{i} b=\lambda^{i} \partial_{i} a, \partial_{i} e=\zeta^{i} \partial_{i} a
$$

along with the dispersion relation

$$
\zeta^{i} \lambda^{i}=Q_{a}+Q_{b} \lambda^{i}+Q_{e} \zeta^{i}
$$

As before, the commutativity conditions (7) lead to the expressions for $\partial_{j} \lambda^{i},(i \neq j)$, and the compatibility conditions of the equations $\partial_{i} b=\lambda^{i} \partial_{i} a, \partial_{i} e=\zeta^{i} \partial_{i} a$ yield (9). The consistency conditions $\partial_{k} \partial_{j} \lambda^{i}-\partial_{j} \partial_{k} \lambda^{i}=0$ are of the form $R \partial_{j} a \partial_{k} a=0$ where $R$ is a rational expression in $\lambda^{i}, \lambda^{j}, \lambda^{k}$ whose coefficients depend on partial derivatives of $Q(e, a, b)$ up to the third order. Requiring that $R$ vanishes identically we obtain the expressions for all third order partial derivatives of $Q$. Similarly, the compatibility conditions of the equations (9), that is, $\partial_{k}\left(\partial_{i} \partial_{j} a\right)-\partial_{j}\left(\partial_{i} \partial_{k} a\right)=0$, take the form $M \partial_{i} a \partial_{j} a \partial_{k} a=0$ where, 
again, $M$ is a rational expression in $\lambda^{i}, \lambda^{j}, \lambda^{k}$. Equating $M$ to zero one obtains exactly the same conditions as on the previous step. The final set of integrability conditions looks as follows:

$$
\begin{aligned}
& Q_{b b b}= \frac{Q_{b b}\left(Q_{b} Q_{b e}+Q_{e} Q_{b b}+Q_{a b}\right)}{Q_{b} Q_{e}+Q_{a}}, \quad Q_{e e e}=\frac{Q_{e e}\left(Q_{e e} Q_{b}+Q_{e} Q_{b e}+Q_{a e}\right)}{Q_{b} Q_{e}+Q_{a}} \\
& Q_{b b e}= \frac{Q_{b b}\left(Q_{e e} Q_{b}+Q_{e} Q_{b e}+Q_{a e}\right)}{Q_{b} Q_{e}+Q_{a}}, \quad Q_{b e e}=\frac{Q_{e e}\left(Q_{b} Q_{b e}+Q_{e} Q_{b b}+Q_{a b}\right)}{Q_{b} Q_{e}+Q_{a}} \\
& Q_{a e e}= \frac{Q_{e e}\left(Q_{b} Q_{a e}+Q_{e} Q_{a b}+Q_{a a}\right)}{Q_{b} Q_{e}+Q_{a}}, \quad Q_{a b b}=\frac{Q_{b b}\left(Q_{b} Q_{a e}+Q_{e} Q_{a b}+Q_{a a}\right)}{Q_{b} Q_{e}+Q_{a}} \\
& Q_{a a b}= \frac{Q_{a b}\left(2 Q_{e} Q_{a b}+Q_{a a}\right)+Q_{b b}\left(2 Q_{a} Q_{a e}-Q_{e} Q_{a a}\right)-Q_{b e}\left(2 Q_{a} Q_{a b}-Q_{b} Q_{a a}\right)}{Q_{b} Q_{e}+Q_{a}} \\
& Q_{a b e}=\frac{Q_{a e} Q_{a b}+Q_{a}\left(Q_{b b} Q_{e e}-Q_{b e}^{2}\right)+Q_{b e}\left(Q_{b} Q_{a e}+Q_{e} Q_{a b}\right)}{Q_{b} Q_{e}+Q_{a}} \\
& Q_{a a e}= \frac{2 Q_{a} Q_{a b} Q_{e e}+Q_{a a} Q_{a e}+Q_{b e}\left(Q_{e} Q_{a a}-2 Q_{a} Q_{a e}\right)+Q_{b}\left(2 Q_{a e}^{2}-Q_{a a} Q_{e e}\right)}{Q_{b} Q_{e}+Q_{a}} \\
& Q_{a a a}=\left(Q_{a a}\left[2\left(Q_{b}^{2} Q_{e e}+Q_{e}^{2} Q_{b b}\right)+Q_{b} Q_{a e}+Q_{e} Q_{a b}+Q_{a a}-2 Q_{b e}\left(Q_{a}+2 Q_{b} Q_{e}\right)\right]\right. \\
&+2 Q_{a b}\left[Q_{a}\left(Q_{a e}+2 Q_{e} Q_{b e}\right)+2 Q_{b}\left(Q_{e} Q_{a e}-Q_{a} Q_{e e}\right)-Q_{e}^{2} Q_{a b}\right] \\
&\left.+2 Q_{a e}\left[Q_{a}\left(2 Q_{b} Q_{b e}-2 Q_{e} Q_{b b}\right)-Q_{b}^{2} Q_{a e}\right]+2 Q_{a}^{2}\left[Q_{b b} Q_{e e}-Q_{b e}^{2}\right]\right) /\left(Q_{b} Q_{e}+Q_{a}\right)
\end{aligned}
$$

This system is in involution and its general solution depends on 10 integration constants.

It is easy to see that the general solution of the first six equations is

$$
Q=\frac{1}{4} \ln \frac{U(a, p)}{V(a, q)}, \quad 4 U_{a}=U_{p p}, \quad 4 V_{a}=V_{q q}
$$

where

$$
p=b-e, \quad q=b+e .
$$

The general solution of the system (14) can be obtained by the substitution of this ansatz into the remaining equations. Let us introduce the notation

$$
Q_{p}=-\frac{1}{4} k, \quad Q_{p p}=-\frac{1}{4} l, \quad Q_{q}=\frac{1}{4} m, \quad Q_{q q}=\frac{1}{4} n,
$$

here $\partial_{b}=\partial_{q}+\partial_{p}, \quad \partial_{e}=\partial_{q}-\partial_{p}$. Then the equation $(14)_{8}$ takes the form

$$
4 n n_{a}-n_{q}\left(n_{q}-2 m n\right)+2 n\left[2 l_{a}+k l_{p}-2 l^{2}\right]=4 l l_{a}-l_{p}\left(l_{p}-2 k l\right)+2 l\left[2 n_{a}+m n_{q}-2 n^{2}\right]
$$

where

$$
\begin{aligned}
Q_{a} & =\frac{1}{16}\left[-l+n+k^{2}-m^{2}\right], \quad Q_{b e}=\frac{1}{4}(n+l), \quad Q_{b}=\frac{1}{4}(m-k), \\
Q_{e} & =\frac{1}{4}(m+k), \quad Q_{b b}=\frac{1}{4}(n-l), \quad Q_{e e}=\frac{1}{4}(n-l), \\
Q_{a e} & =\frac{1}{16}\left[n_{q}-2 m n+l_{p}-2 k l\right], \quad Q_{a b}=\frac{1}{16}\left[n_{q}-2 m n-l_{p}+2 k l\right] .
\end{aligned}
$$

The differentiation of (15) twice with respect to $p$ and $q$ implies

$$
n_{q}\left[2 l_{a}+k l_{p}-2 l^{2}\right]_{p}=l_{p}\left[2 n_{a}+m n_{q}-2 n^{2}\right]_{q} .
$$


Assuming that $n_{q} \neq 0$ and $l_{p} \neq 0$ one obtains

$$
l_{a}=l^{2}-\frac{1}{2} k l_{p}-\eta(a) l-C(a), \quad n_{a}=n^{2}-\frac{1}{2} m n_{q}-\eta(a) n-B(a),
$$

where $\eta(a), B(a)$ and $C(a)$ are functions to be determined. Substituting $n_{a}$ and $l_{a}$ into (15) one has

$l_{p}^{2}=4 l^{3}-4 \eta(a) l^{2}-4[B(a)+C(a)] l-E(a), \quad n_{q}^{2}=4 n^{3}-4 \eta(a) n^{2}-4[B(a)+C(a)] n-E(a)$, where $E(a)$ is yet another undetermined function. Checking the compatibility conditions $\left(l_{p}\right)_{a}=\left(l_{a}\right)_{p}, \quad\left(n_{q}\right)_{a}=\left(n_{a}\right)_{q}$ one obtains

$$
B=C=\eta^{\prime}, \quad E=\frac{8}{3} \eta^{\prime \prime},
$$

where $\eta$ is a solution of the Chazy equation (2).With these formulas the remaining expressions for $Q_{a a a}, Q_{a a b}$ and $Q_{a a c}$ hold identically.

Thus, the general solution of the involutive system (14) yields the main classification result of this section:

$$
\Omega_{z t}=\frac{1}{4} \ln \frac{\theta_{1}\left(\Omega_{x x}, \Omega_{x t}-\Omega_{x z}\right)}{\theta_{1}\left(\Omega_{x x}, \Omega_{x t}+\Omega_{x z}\right)}
$$

where $\theta_{1}$ is the Jacobi theta-function defined by (3).

Remark: This formula provides a pseudopotential for the general case

$$
\Omega_{t t}=\ln \Omega_{x y}-\ln \theta_{1}\left(\Omega_{x t}, \Omega_{x x}\right)-\frac{1}{4} \int^{\Omega_{x x}} \eta(\tau) d \tau,
$$

see the Introduction. All other particular cases can be obtained by appropriate degenerations, see [2].

Remark: Under linear transformation of independent variables $(z, t)$ this equation can be written in more symmetric form

$$
e^{\Omega_{z z}} \theta_{1}\left(\Omega_{x x}, \Omega_{x z}\right)=e^{\Omega_{t t}} \theta_{1}\left(\Omega_{x x}, \Omega_{x t}\right) .
$$

To calculate $L$ we consider the compatibility condition

$$
\partial_{z} G(a, b, c)=\partial_{t} Q(e, a, b)
$$

which implies

$$
G_{a} e_{x}+G_{b} Q(a, b, e)_{x}+G_{c} L(e, a, b, c)_{x}=Q_{e} Q(a, b, e)_{x}+Q_{a} b_{x}+Q_{b} G(a, b, c)_{x} ;
$$

recall that $c_{z}=L(e, a, b, c)_{x}$. Therefore,

$$
L=Q_{b} c+f(e, a, b) .
$$

along with

$$
\begin{aligned}
Q_{b b} & =\varepsilon\left(Q_{a}+Q_{b} Q_{e}\right), \quad Q_{a b}=\varepsilon\left(Q_{a} Q_{e}+Q_{b} F_{a}-F_{b} Q_{a}\right) \\
Q_{b e} & =\varepsilon\left(Q_{e}^{2}-F_{b} Q_{e}-F_{a}\right), \quad f_{b}=(\alpha a+\beta b+\gamma)\left(Q_{a}+Q_{b} Q_{e}\right), \\
f_{a} & =(\alpha a+\beta b+\gamma)\left(Q_{a} Q_{e}+Q_{b} F_{a}-F_{b} Q_{a}\right)+\frac{1}{\varepsilon}\left(\alpha Q_{b}-\beta Q_{a}\right), \\
f_{e} & =(\alpha a+\beta b+\gamma)\left(Q_{e}^{2}-F_{b} Q_{e}-F_{a}\right)-\frac{1}{\varepsilon}\left(\beta Q_{e}+\alpha\right) .
\end{aligned}
$$


These relations are sufficient for the reconstruction of $L$.

\section{Conclusion}

We have proved that the method of hydrodynamic reductions applied to quasilinear equations (5) and (13) yields the same classification results as the symmetry approach used in [10]. Thus, both methods allow to classify integrable $(2+1)$-dimensional equations and find their commuting flows, see [10] for the details.

\section{Acknowledgements}

We would like to thank the London Mathematical Society for their financial support of MVP to Loughborough, making this collaboration possible.

\section{References}

[1] M. Abramowitz and I.A. Stegun, Handbook of mathematical functions with formulas, graphs, and mathematical tables. John Wiley \& Sons, Inc., New York; National Bureau of Standards, Washington, DC (1984) 1046 pp.

[2] V.M. Buchstaber, D.V. Leikin and M.V. Pavlov, The Egorov hydrodynamic chains, the Chazy equation and $S L(2, C)$ group, Func. Anal. Appl. 37 (2003) 13-26.

[3] C.P. Boyer and J.D. Finley, Killing vectors in self-dual Euclidean Einstein spaces, J. Math. Phys. 23 (1982) 1126-1130.

[4] R. Carroll and Yu. Kodama, Solutions of the dispersionless Hirota equations, J. Phys. A: Math. Gen. 28 (1995) 6373-6387.

[5] J. Chazy, Sur les équations différentiellles dont l'intégrale générale possède un coupure essentielle mobile, C.R. Acad. Sc. Paris, 150 (1910) 456-458.

[6] E.V. Ferapontov and K.R. Khusnutdinova, On the integrability of (2+1)-dimensional quasilinear systems, Comm. Math. Phys. 248 (2004) 187-206; E.V. Ferapontov and K.R. Khusnutdinova, The characterization of two-component (2+1)-dimensional integrable systems of hydrodynamic type, J. Phys. A: Math. Gen. 37 (2004) 2949-2963; E.V. Ferapontov and K.R. Khusnutdinova, Hydrodynamic reductions of multi-dimensional dispersionless PDEs: the test for integrability, J. Math. Phys. $\mathbf{4 5}$ (2004) 2365-2377.

[7] J. Gibbons and S.P. Tsarev, Reductions of the Benney equations, Phys. Lett. A 211 (1996)19-24; J. Gibbons and S.P. Tsarev, Conformal maps and reductions of the Benney equations, Phys. Lett. A 258 (1999) 263-270.

[8] I.M. Krichever, A. Marshakov and A. Zabrodin, Integrable structure of the Dirichlet boundary problem in multiply-connected domains. arXiv:hep-th/0309010.

[9] M. Manas, L. M. Alonso and E. Medina, Reductions and hodograph solutions of the dispersionless KP hierarchy, J. Phys. A: Math. Gen. 35 (2002) 401-417.

[10] M.V. Pavlov, New integrable (2+1)-equations of hydrodynamic type, Russian Math. Surv., 58, No. 2 (2003) 384-385; M.V. Pavlov, Classifying integrable Egoroff hydrodynamic chains, Theor. Math. Phys. 138 (2004) 45-58.

[11] S.P. Tsarev, Poisson brackets and one-dimensional Hamiltonian systems of hydrodynamic type, Soviet Math. Dokl. 31 (1985) 488-491; S.P. Tsarev, The geometry of Hamiltonian systems of hydrodynamic type. The generalized hodograph method, Math. USSR Izvestiya 37 (1991) 397-419.

[12] E.V. Zakharov, Dispersionless limit of integrable systems in $2+1$ dimensions, in Singular Limits of Dispersive Waves, Ed. N.M. Ercolani et al., Plenum Press, NY (1994) 165-174. 